\title{
Estudo comparativo entre ressecção do trapézio e interposição tendinosa com e sem ligamentoplastia no tratamento da artrose carpometacarpiana do polegar*
}

\author{
Comparative study between trapezium resection and tendon \\ interposition with and without ligament plasty in the \\ management of carpometacarpal arthrosis of the thumb
}

\author{
Arlindo Gomes Pardini JUNIOR ${ }^{1}$, Afrânio Donato de Freitas ${ }^{2}$, \\ Antonio Barbosa Chaves ${ }^{3}$, MARCel Barbieri Freitas ${ }^{4}$
}

\section{RESUMO}

Objetivos: $O$ tratamento da artrose da base do polegar tem sido tema de grande controvérsia. Numerosas cirurgias têm sido descritas, como ressecção isolada do trapézio, ressecção com interposição com e sem reforço ligamentar, artrodese e artroplastias. $O$ objetivo deste trabalho foi o de comparar os resultados das duas técnicas mais utilizadas nesse tratamento. Métodos: Foi realizado estudo prospectivo a fim de comparar os resultados cirúrgicos de ressecção do trapézio com interposição tendinosa (tenoartroplastia) - 22 casos e tenoartroplastia associada a ligamentoplastia - 24 ca-

* Trabalho realizado no Hospital Ortopédico de Belo Horizonte, Belo Horizonte (MG), Brasil.

1. Livre-Docente em Ortopedia e Traumatologia; Chefe do Serviço de Cirurgia da Mão do Hospital Ortopédico de Belo Horizonte, Belo Horizonte (MG), Brasil.

2. Chefe do Serviço de Cirurgia da Mão do Hospital Belo Horizonte, Belo Horizonte (MG), Brasil.

3. Cirurgião de Mão dos Hospitais Ortopédico de Belo Horizonte e Maria Amélia Lins, Belo Horizonte (MG), Brasil.

4. Residente de Cirurgia da Mão do Hospital Ortopédico de Belo Horizonte, Belo Horizonte (MG), Brasil.

Endereço para correspondência: Arlindo G. Pardini Jr., Rua Prof. Otavio Coelho de Magalhães, 111 - 30210-300 - Belo Horizonte, MG. Tel.: (31) 3289-1236; fax: (31) 3227-0666.

E-mail: centrodeestudosho@hotmail.com

Recebido em 14/1/08. Aprovado para publicação em 4/3/08.

Copyright RBO2008
Sos. A avaliação objetiva foi feita através das medidas de oponência, dos movimentos da articulação metacarpofalangiana, da força de pinça e de preensão, da medida radiográfica da distância entre a base do 1 metacarpiano e o escafóide e do ângulo entre o $1^{\circ}$ e $02^{\circ}$ metacarpianos. A avaliação subjetiva foi realizada através do questionário DASH, uma escala analógico-digital para avaliar a dor e a satisfação do paciente. Resultados e conclusão: Após a aplicação dos critérios descritos e usada análise estatística pelo teste $t$ de Student, os autores concluem não haver vantagens da reconstrução ligamentar sobre a simples interposição tendinosa na artrose carpometacarpiana do polegar.

Descritores - Osteoartrite; Trapézio/cirurgia; Polegar/patologia; Doenças músculo-esqueléticas/cirurgia; Artroplastia/métodos; Estudos comparativos

\section{ABSTRACT}

Objectives: Treating arthrosis in the base of the thumb has been a highly controverted subject. Many surgeries have been described, such as the isolated trapezium resection; resection with interposition with and without ligament plasty, arthrodesis, and arthroplasties. The purpose of this paper is to compare the two techniques which are the most used in this treatment. Methods: A prospective study has been made to compare the surgical results between 
the trapezium resection with tendon interposition (tendon arthroplasty) - 22 cases - and tendon arthroplasty associated to ligament plasty - 24 cases. Objective evaluation was done by measuring opponency, movements of the metacarpophalangeal joint, pinching and pressure strength, radiographic measurement of the distance between the first metacarpian and the scaphoid, and measurement of the angle between the first and the second metacarpian. Subjective evaluation was done with a DASH questionnaire, an analogical-digital scale to evaluate pain and patient satisfaction. Results and conclusion: After application of the criteria described and using Student $t$ test for statistical analysis, the authors concluded that the ligament reconstruction has no advantage over the simple tendon interposition in carpometacarpal arthrosis of the thumb.

Keywords - Osteoarthritis; Trapezium bone/surgery; Thumb/pathology; Musculoskeletal diseases/ surgery; Arthroplasty/methods; Comparative studies

\section{INTRODUÇÃO}

A osteoartrose trapézio- $1^{\circ}$ metacarpiano ou rizartrose é das afecções articulares degenerativas mais freqüentes na mão e afeta, principalmente, mulheres após a menopausa. Nem todos os pacientes portadores de artrose da base do polegar apresentam dor, porém, quando esta é intensa, afetando as atividades normais e é resistente aos tratamentos conservadores, existe indicação cirúrgica.

Inúmeras técnicas cirúrgicas têm sido publicadas, todas elas apresentando resultados satisfatórios. A simples ressecção do trapézio foi descrita por Gervis, em $1949^{(1)}$. Ele ficou tão impressionado com o resultado, que realizou essa cirurgia em si mesmo, quando teve a rizartrose (apud Davis TR, et al) ${ }^{(2)}$. Posteriormente, outros autores relataram também bons resultados com essa cirurgia ${ }^{(3-7)}$. Devido à possível migração proximal e instabilidade, causadas pela ressecção do trapézio, que poderia, pelo menos teoricamente, ocasionar problemas à nova articulação, foram introduzidas outras técnicas com a finalidade de estabilizar a base do
1ำ metacarpiano através de reconstruções ligamentares com ou sem interposição de material biológico ou não ${ }^{(8-12)}$. Autores recomendam a ressecção do trapézio e apenas interposição tendinosa sem reconstrução ligamentar $^{(13-16)}$. No entanto, a cirurgia que parece ser a preferida ultimamente é a combinação da trapeziectomia com ligamentoplastia e interposição tendinosa, embora muitos autores concordem com o fato de que a simples ressecção do trapézio dá os mesmos resultados que a associação com aquelas técnicas ${ }^{(2,17-21)}$.

Artrodese trapézio-1 metacarpiano para tratamento da artrose desta articulação tem sido abandonada devido à limitação de movimento resultante e à deformidade em hiperextensão da metacarpofalangiana (MF), embora possa levar ao alívio da dor e melhora da força ${ }^{(22-25)}$.

As artroplastias de substituição com próteses tipo Swanson ou Caffinière, embora se relatem bons resultados, têm sido abandonadas devido à grande incidência de complicações, à complexidade do método e ao alto custo dos implantes ${ }^{(26-29)}$.

A finalidade deste trabalho é comparar os resultados da tenoartroplastia com e sem ligamentoplastia, numa tentativa de apontar a melhor opção a adotar na indicação do tratamento cirúrgico da rizartrose do polegar.

\section{MÉTODOS}

Em 1995, um dos autores avaliou os resultados da artrodese, ligamentoplastia e tenoartroplastia no tratamento da rizartrose ${ }^{(23)}$. A partir desse trabalho, o estudo foi padronizado no Serviço de Cirurgia de Mão do Hospital Belo Horizonte-MG, de tal forma que um cirurgião realizou apenas tenoartroplastia com interposição de uma fita do tendão flexor radial do carpo pela técnica de Froimson ${ }^{(16)}$ e outros dois cirurgiões procederam à ressecção do trapézio seguida de reconstrução ligamentar com uma fita do tendão longo abdutor do polegar (pela técnica de Thompson) $)^{(12)}$; os resultados foram avaliados por um examinador que não participou daquelas cirurgias. O estudo teve a aprovação do Comitê de Ética do Hospital de Belo Horizonte-MG. De fevereiro de 1996 a novembro de 2006, foram operados 82 pacientes. Foram excluídos do estudo 
pacientes do sexo masculino que poderiam afetar a homogeneidade do grupo.

Todas as cirurgias foram realizadas sob bloqueio anestésico do plexo braquial e torniquete pneumático.

Entre os pacientes, 41 compareceram para a avaliação, correspondendo a 46 mãos operadas; duas pacientes do grupo I e três do grupo II foram operadas bilateralmente. Todas tinham rizartrose classificada nos tipos II $(9,5 \%)$, III $(57,1 \%)$ e IV $(33,3 \%)$ de Eaton e Glieckel e 14 haviam sido submetidas previamente a tratamento conservador ${ }^{(30)}$.

Das pacientes avaliadas, 20 (22 mãos) foram submetidas à ressecção do trapézio e interposição da metade do flexor radial do carpo (grupo I) e em 21 (24 mãos) foi realizada a ligamentoplastia com um tendão do abdutor longo do polegar, além da ressecção do trapézio (grupo II) (tabela 1).

O tratamento pós-operatório foi o mesmo nos dois grupos, isto é, imobilização com tala gessada curta incluindo o polegar, por três a quatro semanas e, após esse período, eram encaminhados à fisioterapia.

A média de idade das pacientes foi de 67,19 anos (mínima de 48 e máxima de 82), sendo semelhante

TABELA 1

Distribuição do número de casos conforme técnica cirúrgica empregada

\begin{tabular}{lcc}
\hline \multicolumn{1}{c}{ Técnica cirúrgica } & Número de casos & $\%$ \\
\hline Tenoartroplastia & 22 & 47,8 \\
Tenoartroplastia + ligamentoplastia & 24 & 52,2 \\
Total & 46 & 100 \\
\hline
\end{tabular}

Fonte: SAME Hospital Ortopédico, Belo Horizonte-MG. nos dois grupos. A tabela 2 mostra a freqüência conforme a idade, tempo de seguimento e tempo de início dos sintomas.

Entre as pacientes, duas haviam sido submetidas a procedimentos prévios, uma tentativa de artrodese carpometacarpiana no grupo I e uma tenotomia do abdutor longo do polegar no grupo II. Três pacientes do grupo I foram submetidas a procedimentos complementares, uma artrodese metacarpofalangiana, uma capsulodese metacarpofalangiana; em uma paciente essa articulação foi fixada por três semanas com fio de Kirschner após a trapeziectomia (mesma paciente que fora submetida a artrodese contralateral). No grupo II não houve cirurgias complementares.

Os métodos de avaliação foram: mobilidade demonstrada pela capacidade de opor o polegar à polpa do quinto dedo; flexão e extensão máximas da articulação metacarpofalangiana; força de preensão, força de pinça de polpa e de chave; medida radiográfica da distância do escafóide à base do $1^{\circ}$ metacarpiano e o ângulo entre o $1^{\circ}$ e $2^{\circ}$ metacarpianos.

$\mathrm{Na}$ avaliação subjetiva foi usado o questionário DASH (Disability Arm, Shoulder and Hand), uma escala analógico-digital para avaliar a dor pré e pós-operatória e foi pedido às pacientes para classificar sua satisfação com relação ao resultado da cirurgia.

$\mathrm{Na}$ análise estatística dos dados utilizou-se o teste $t$ de Student, sendo considerados significantes os valores de $\mathrm{p}<0,05$. Através de programas de processamento estatístico (Epi Info v. 6.04d) foram construídas distribuições de freqüências e calculados as médias, desvio-padrão e percentagens.

TABELA 2

Distribuição das freqüências conforme idade, tempo de seguimento, início dos sintomas e técnica cirúrgica aplicada

\begin{tabular}{|c|c|c|c|c|c|c|c|c|}
\hline \multirow[t]{2}{*}{ Técnica cirúrgica } & \multicolumn{4}{|c|}{ Tenoartroplastia } & \multicolumn{4}{|c|}{$\begin{array}{l}\text { Tenoartroplastia + } \\
\text { ligamentoplastia }\end{array}$} \\
\hline & Mín & Máx & Méd & DP & Mín & Máx & Méd & DP \\
\hline Idade (em anos) & 51 & 82 & 68,04 & 7,81 & 48 & 82 & 66,41 & 9,58 \\
\hline Seguimento (em meses) & 9 & 138 & 80,09 & 40,45 & 9 & 128 & 61,56 & 32,67 \\
\hline Início dos sintomas (em meses) & 2 & 84 & 35,19 & 25,21 & 1 & 60 & 15,89 & 17,16 \\
\hline
\end{tabular}

Fonte: SAME Hospital Ortopédico, Belo Horizonte-MG. $\mathrm{DP}=$ Desvio-padrão. 


\section{RESULTADOS}

O tempo de seguimento médio entre a cirurgia e a avaliação dos resultados foi de 70,15 meses (DP = 37,35) com o mínimo de nove meses e o máximo de 138 meses. Esse tempo de follow-up foi semelhante nos dois grupos: de nove a 138 meses no grupo I e de nove a 128 meses no grupo II.

Houve uma complicação pós-operatória em cada grupo, nenhuma delas relacionada ao método cirúrgico (uma plexopatia braquial no grupo I e uma rigidez interfalangiana do polegar no grupo II).

$\mathrm{O}$ resultado com relação à oponência do polegar foi bom nas pacientes dos dois grupos, pois todas tocavam a ponta do polegar na polpa do dedo mínimo.

A análise da amplitude da flexão e da extensão da articulação metacarpofalangiana (MF) do polegar mostrou no grupo I, submetido a tenoartroplastia, flexão média de $44,62^{\circ}$ (mínima de $20^{\circ}$ e máxima de $74^{\circ}$ ) e no grupo II, da ligamentoplastia, 51,67º (mínima de $18^{\circ}$ e máxima de $80^{\circ}$ ). A extensão média da MF do 1 o grupo foi de $39,05^{\circ}$ (mínima de $0^{\circ}$ e máxima de $90^{\circ}$ ) e no $2^{\circ}$ grupo, de $38,7^{\circ}$ (mínima de $16^{\circ}$ e máxima de $70^{\circ}$ ). Vemos, portanto, que em ambos grupos há tendência à hiperextensão da articulação metacarpofalangiana.

A força de preensão foi medida com o aparelho de JAMAR e as forças de pinça de polpa (ponta) e pinça de chave (lateral) com o pinch meter. Todas essas medidas foram realizadas conforme a técnica padrão, isto é, com o cotovelo a $90^{\circ}$ e sem apoiar o membro superior na mesa.
A força de preensão no grupo I foi em média de 21,07kg/f (mínima de 12,33 e máxima de 36,0). No grupo II a média foi de $22,11 \mathrm{~kg} / \mathrm{f}$ (mínima de $10^{\circ} \mathrm{e}$ máxima de 28,66).

A força da pinça de polpa no grupo I foi em média de $5,09 \mathrm{~kg} / \mathrm{f}$ (mínima de 2,41 e máxima de 9,53). No grupo II a média foi de 5,06kg/f (mínima de 2,91 e máxima de 7,20).

A força da pinça de chave no grupo I foi em média de 4,79kg/f (mínima de 2,05 e máxima de 8,63). No grupo II a média foi de 4,63kg/f (mínima de 2,00 e máxima de 7,23).

A tabela 3 mostra as médias da amplitude de movimentos (ADM) da metacarpofalangiana (MF), a força conforme a técnica cirúrgica empregada, e o desviopadrão (DP).

A distância entre o escafóide e a base do 1o metacarpiano foi medida em radiografias padronizadas na incidência em perfil e não evidenciou grande diferença entre as duas técnicas cirúrgicas. No grupo I a distância média foi de 3,88mm (mínima de 1,00 e máxima de 6,00) e no grupo II, de 3,18mm (mínima de 1,00 e máxima de 6,00). A medida do ângulo de abdução, entre o eixo do $1^{\circ}$ e do $2^{\circ}$ metacarpianos, foi também padronizada com radiografias na incidência pósteroanterior em abdução máxima ativa. Estas foram as seguintes: no grupo I, a média do ângulo de abdução foi de $35,36^{\circ}$ (mínimo de $15,00^{\circ}$ e máximo de $57,00^{\circ}$ ) e no grupo II, de $37,04^{\circ}$ (mínimo de $14,00^{\circ}$ e máximo de $\left.60,00^{\circ}\right)$.

TABELA 3

Distribuição das médias de ADM e força por técnica cirúrgica aplicada

\begin{tabular}{|c|c|c|c|c|c|c|c|c|}
\hline \multirow[t]{2}{*}{ Técnica cirúrgica } & \multicolumn{4}{|c|}{ Tenoartroplastia } & \multicolumn{4}{|c|}{$\begin{array}{l}\text { Tenoartroplastia + } \\
\text { ligamentoplastia }\end{array}$} \\
\hline & Mín & Máx & Méd & DP & Mín & Máx & Méd & DP \\
\hline Flexão MF & $20,00^{\circ}$ & $74,00^{\circ}$ & $44,62^{\circ}$ & 16,63 & $18,00^{\circ}$ & $80,00^{\circ}$ & $51,67^{\circ}$ & 14,03 \\
\hline Extensão MF & $0,00^{\circ}$ & $90,00^{\circ}$ & $39,05^{\circ}$ & 23,11 & $16,00^{\circ}$ & $70,00^{\circ}$ & $38,71^{\circ}$ & 13,50 \\
\hline Pinça de polpa (kg/f) & 2,41 & 9,53 & 5,09 & 1,69 & 2,91 & 7,20 & 5,06 & 1,24 \\
\hline Pinça de chave $(\mathrm{kg} / \mathrm{f})$ & 2,05 & 8,63 & 4,79 & 1,71 & 2,00 & 7,23 & 4,63 & 1,24 \\
\hline Preensão (kg/f) & 12,33 & 36,00 & 21,07 & 5,37 & 10,00 & 28,66 & 22,11 & 4,26 \\
\hline
\end{tabular}

Fonte: SAME Hospital Ortopédico, Belo Horizonte-MG. DP = Desvio-padrão. 
TABELA 4

Distribuição da medida da distância esc-met e ângulo I - II M por técnica cirúrgica aplicada

\begin{tabular}{|c|c|c|c|c|c|c|c|c|}
\hline \multirow[t]{2}{*}{ Técnica cirúrgica } & \multicolumn{4}{|c|}{ Tenoartroplastia } & \multicolumn{4}{|c|}{$\begin{array}{l}\text { Tenoartroplastia + } \\
\text { ligamentoplastia }\end{array}$} \\
\hline & Mín & Máx & Méd & DP & Mín & Máx & Méd & DP \\
\hline Esc-met (em mm) & 1,00 & 6,00 & 3,88 & 1,41 & 1,00 & 6,00 & 3,18 & 1,13 \\
\hline Ângulo I - II M & $15,00^{\circ}$ & $57,00^{\circ}$ & $35,36^{\circ}$ & 10,18 & $14,00^{\circ}$ & $60,00^{\circ}$ & $37,04^{\circ}$ & 11,87 \\
\hline
\end{tabular}

Fonte: SAME Hospital Ortopédico, Belo Horizonte-MG

$\mathrm{DP}=$ Desvio-padrão.

Na tabela 4 está a descrição das medidas da distância do escafóide à base do 1 o metacarpiano (esc-met) e do ângulo 1으ㄴㅡㅜ metacarpianos (I - II M).

A avaliação da dor foi feita por meio de uma escala analógico-digital, em que, numa régua, o paciente apontava o grau de dor de 0 a 10 . Nos pacientes do grupo I a média de dor pré-operatória foi de 7,7 (mínima de 3,0 e máxima de 10,0). Na avaliação pós-operatória, a média foi de 1,1 (mínima de 0,0 e máxima de 7,2). A paciente de pior resultado foi a que teve uma plexopatia braquial. Quanto aos pacientes do grupo II, a dor pré-operatória era em média de 8,2 (mínima de $5,6$ e máxima de 10,0$)$ e após a cirurgia a média era de 0,4 (mínima de 0,0 e máxima de 2,9). O percentual de melhora da dor no grupo I foi de $86,4 \%$ e no grupo II, de $95,9 \%$.

A avaliação subjetiva dos resultados tem hoje um método comprovado de análise, que é o questionário DASH (Disabilities of the Arm, Shoulder and Hand), em que o paciente responde a 34 itens relacionados à sua capacidade funcional e a restrições a atividades de lazer e de trabalho ${ }^{(31)}$. As respostas a cada item têm valor de 1 a 5 ( 1 = nenhuma dificuldade; 2 = pequena dificuldade; 3 = dificuldade moderada; $4=$ grande dificuldade; $5=$ incapaz). Isso produz uma escala de 0 a 100 , em que o valor maior indica maior incapacidade. Os resultados das cirurgias avaliados pelo DASH mostraram no grupo I média de 12,5 (mínima de 0,0 e máxima de 55,8 ), enquanto que no grupo II a média foi de 11,1 (mínima de 0,0 e máxima de 50,9).

A tabela 5 mostra a distribuição da dor pré e pósoperatória e os resultados do DASH conforme a técnica cirúrgica.

Com relação à satisfação pessoal, solicitamos às pacientes informar sua satisfação em relação ao resultado cirúrgico, entre as seguintes opções: muito satisfeita, satisfeita, pouco satisfeita e insatisfeita. No grupo I, $63,6 \%$ das pacientes estavam muito satisfeitas, $27,3 \%$ satisfeitas e $9,1 \%$ insatisfeitas. Entre estas, uma foi devido à plexopatia braquial em decorrência da

TABELA 5

Distribuição das aferições de dor pré e pós-operatória, média percentual de melhora e escala DASH por técnica cirúrgica aplicada

\begin{tabular}{|c|c|c|c|c|c|c|c|c|c|c|}
\hline \multirow[t]{2}{*}{ Técnica cirúrgica } & \multicolumn{5}{|c|}{ Tenoartroplastia } & \multicolumn{5}{|c|}{$\begin{array}{c}\text { Tenoartroplastia + } \\
\text { ligamentoplastia }\end{array}$} \\
\hline & Mín & Máx & Méd & DP & $\mathbf{p}$ & Mín & Máx & Méd & DP & $\mathbf{p}$ \\
\hline Dor pré-operatória & 3,00 & 10,0 & 7,7 & 2,05 & 0,00 & 5,6 & 10,0 & 8,2 & 1,40 & 0,00 \\
\hline Dor pós-operatória & 0,0 & 7,2 & 1,1 & 2,05 & 0,02 & 0,0 & 2,9 & 0,4 & 0,76 & 0,03 \\
\hline$\%$ de melhora & 0,0 & 100 & 86,4 & 26,7 & 0,00 & 65 & 100 & 95,9 & 8,91 & 0,00 \\
\hline DASH & 0,0 & 55,8 & 12,5 & 16,7 & 0,00 & 0,0 & 50,9 & 11,1 & 12,5 & 0,00 \\
\hline
\end{tabular}

Fonte: SAME Hospital Ortopédico, Belo Horizonte-MG.

$\mathrm{DP}=$ Desvio-padrão. 
anestesia e a outra estava insatisfeita com a hiperextensão desenvolvida na articulação metacarpofalangiana.

No grupo II, $75 \%$ das pacientes estavam muito satisfeitas e $25 \%$ satisfeitas com o resultado da cirurgia.

\section{DISCUSSÃO}

Existe ainda muita controvérsia na literatura com relação à melhor indicação cirúrgica para o tratamento da osteoartrose da base do polegar. No entanto, a maioria dos autores concorda que a ressecção do trapézio é uma parte importante para o alívio da dor. $\mathrm{O}$ tratamento ideal seria aquele que, além do alívio da dor, proporcionasse estabilidade articular com movimento e força. A simples ressecção do trapézio é relatada por poucos autores, como um possível risco de artrose entre as superfícies incongruentes da base do 1을 metacarpiano e do escafóide ${ }^{(32)}$. No entanto, estudos pós-operatórios de seguimentos a longo prazo não demonstraram essa complicação ${ }^{(3-4,6)}$ embora Iyer tenha observado quistos na base do $1^{\circ}$ metacarpiano em $60 \%$ de seus casos, que não influenciaram no resultado final ${ }^{(7)}$. Não observamos essas alterações nas nossas pacientes, mesmo nas operadas havia mais de 10 anos.

Baseados na premissa da possibilidade de migração proximal do 1o metacarpiano e possível instabilidade da base do polegar, vários métodos de reconstrução ligamentar foram descritos, embora não exista um estudo comprovando essa instabilidade. Estudos comparativos entre a trapeziectomia com e sem ligamentoplastia sugerem que não há benefícios de uma técnica sobre outra ${ }^{(2-3,20-21)}$. Embora vários recursos tenham sido tentados para evitar a migração do 1 metacarpiano após a remoção do trapézio, como a ligamentoplastia ou suspensão, fixação com fios de Kirschner ou imobilização pós-operatória prolongada, nenhum deles manteve a altura do espaço original entre a base do $1^{\circ}$ 을 metacarpiano e o escafóide. Na maioria delas ocorreu redução de $50 \%$ a $60 \%$ da dimensão pré-operatória do trapézio. Essa redução do espaço não influenciou no resultado final na análise dos casos com ou sem ligamentoplastia.

Uma das justificativas teóricas de que a remoção do trapézio sem a ligamentoplastia enfraqueceria e tor- naria o polegar instável não foi demonstrada neste estudo, pois não ocorreu diferença significativa entre os dois grupos analisados, com relação às forças de preensão e de pinça. Esse mesmo achado foi observado por Field et al quando compararam o resultado da trapeziectomia simples com a trapeziectomia e ligamentoplastia $^{(20)}$, embora o tempo de seguimento de seus pacientes tenha sido de apenas 12 meses. Também Davis et al não acharam diferença significativa de força de preensão e de pinça quando compararam três grupos de tratamento: trapeziectomia simples, trapeziectomia com interposição tendinosa e trapeziectomia com ligamentoplastia. No entanto, no seu estudo o tempo de seguimento foi de apenas 12 meses $^{(2)}$ comparado com nosso estudo, que foi em média superior a cinco anos.

A maioria das técnicas descritas relata $80 \%$ ou mais de alívio da dor. No entanto, não encontramos na literatura a aplicação do questionário DASH na avaliação subjetiva dos resultados das cirurgias para rizartrose. Os resultados foram muito satisfatórios nos dois grupos, equiparando-se aos das avaliações objetivas.

Não encontramos um trabalho que analisasse os resultados de cirurgias para rizartrose com tantas variáveis como esta. Também o tempo de seguimento longo (de nove a 138 meses), com média de 80 meses para as tenoartroplastias e 61 meses para as tenoartroplastias associadas com ligamentoplastia, foi bastante razoável para reforçar o resultado das duas técnicas cirúrgicas. Confirmamos, assim, que a adição da ligamentoplastia na cirurgia de trapeziectomia e interposição tendinosa não apresenta vantagens apreciáveis.

\section{AGRADECIMENTOS}

Os autores agradecem ao Dr. Saulo Garzedim Freire pela avaliação estatística deste trabalho.

\section{REFERÊNCIAS}

1. Gervis WH. Excision of the trapezium for osteoarthritis of the trapeziometacarpal joint. J Bone Joint Surg Br. 1949;31:537-9.

2. Davis TR, Brady O, Dias JJ. Excision of the trapezium for osteoarthritis of the trapeziometacarpal joint: a study of the benefit of ligament reconstruction or tendon interposition. J Hand Surg [Am]. 2004;29(6):1069-77.

3. De Smet L, Sioen W, Spaepen D, van Rausbeeck H. Treatment of basal joint arthritis of the thumb: trapeziectomy with or 
without tendon interposition/ligament reconstruction. Hand Surg. 2004;9(1):5-9.

4. Varley GW, Calvey J, Hunter JB, Barton NJ, Davis TR. Excision of the trapezium for osteoarthritis at the base of the thumb. J Bone Joint Surg Br. 1994;76(6):964-8.

5. Amadio PC. A comparison of fusion, trapeziectomy, and silastic replacement for the treatment of osteoarthritis of the trapeziometacarpal joint. J Hand Surg [Br]. 2005;30(3):331-2; author reply 332 .

6. Gibbons CE, Gosal HS, Choudri AH, Magnussen PA. Trapeziectomy for basal thumb joint osteoarthritis: 3- to 19year follow-up. Int Orthop. 1999;23(4):216-8.

7. Iyer KM. The results of excision of the trapezium. Hand. 1981; 13(3):246-50.

8. Eaton RG, Lane LB, Littler JW, Keyser JJ. Ligament reconstruction for the painful thumb carpometacarpal joint: a long-term assessment. J Hand Surg [Am]. 1984;9(5):692-9.

9. Friedman DM, Eaton RG, Glickel SZ. Long-term results of volar ligament reconstruction for symptomatic basal joint laxity. J Hand Surg [Am]. 2000;25(2):297-304.

10. Rayan GM, Young BT. Ligament reconstruction arthroplasty for trapeziometacarpal arthrosis. J Hand Surg [Am]. 1997; 22(6):1067-76.

11. Illarramendi AA, Boretto JG, Gallucci GL, De Carli P. Trapeziectomy and intermetacarpal ligament reconstruction with the extensor carpi radialis longus for osteoarthritis of the trapeziometacarpal joint: surgical technique and long-term results. J Hand Surg [Am]. 2006;31(8):1315-21. Comment in: J Hand Surg [Am]. 2007;32(5):747; author reply 747.

12. Thompson JS. Suspensionplasty. J Orthop Surg Techn. 1989;4: $1-13$.

13. Eaton RG, Glickel SZ, Littler JW. Tendon interposition arthroplasty for degenerative arthritis of the trapeziometacarpal joint of the thumb. J Hand Surg [Am]. 1985;10(5):645-54.

14. Froimson AI. Tendon interposition arthroplasty of carpometacarpal joint of the thumb. Hand Clin. 1987;3(4):489505 .

15. Weilby A. Tendon interposition arthroplasty of the first carpometacarpal joint. J Hand Surg [Br]. 1988;13(4):421-5.

16. Froimson AI. Tendon arthroplasty of the trapeziometacarpal joint. Clin Orthop Relat Res. 1970;70:191-9.

17. Burton RI, Pellegrini VD Jr. Surgical management of basal joint arthritis of the thumb. Part II. Ligament reconstruction with tendon interposition arthroplasty. J Hand Surg [Am]. 1986;11(3):324-32.

18. Tomaino MM. Ligament reconstruction tendon interposition arthroplasty for basal joint arthritis. Rationale, current technique, and clinical outcome. Hand Clin. 2001;17(2):20721.
19. Tomaino MM, Pellegrini VD Jr, Burton RI. Arthroplasty of the basal joint of the thumb. Long-term follow-up after ligament reconstruction with tendon interposition. J. Bone Joint Surg Am. 1995;77(3):346-55.

20. Field J, Buchanan D. To suspend or not suspend: a randomised single blind trial of simple trapeziectomy versus trapeziectomy and flexor carpi radialis suspension. J Hand Surg Eur Vol. 2007;32(4):462-6.

21. Belcher HJ, Nicholl JE. A comparison of trapeziectomy with and without ligament reconstruction and tendon interposition. $\mathrm{J}$ Hand Surg [Br]. 2000;25(4):350-6. Comment in: J Hand Surg [Br]. 2001;26(4):392.

22. Pardini AG, Lazaroni AP, Tavares KE. Compression arthrodesis of the carpometacarpal joint of the thumb. Hand. 1982;14(3): 291-4.

23. Pardini Junior AG, Villela EA. Osteoartrite carpometacárpica do polegar: estudo comparativo entre artroplastia de interposição tendinosa, artrodese e ligamentoplastia. Rev Bras Ortop. 1995;30(4):219-26.

24. Mureau MA, Rademaker RP, Verhaar JA, Hovius SE. Tendon interposition arthroplasty versus arthrodesis for the treatment of trapeziometacarpal arthritis: a retrospective comparative follow-up study. J Hand Surg [Am]. 2001;26(5):869-76.

25. Hartigan BJ, Stern PJ, Kiefhaber TR. Thumb carpometacarpal osteoarthritis: arthrodesis compared with ligament reconstruction and tendon interposition. J Bone Joint Surg Am. 2001;83-A(10):1470-8. Comment in: J Bone Joint Surg Am. 2002;84-A(7):1275-6; author reply 1276.

26. Swanson AB. Disabling arthritis at the base of the thumb: treatment by resection of the trapezium and flexible (silicone) implant arthroplasty. J Bone Joint Surg Am. 1972;54(3):45671.

27. Albertoni WM, Leite VM, Faloppa F, Galbiatti JA. Prótese total de De la Caffinière no tratamento da rizartrose do polegar. Rev Bras Ortop. 1992;27(8):581-6.

28. Badia A, Sambandam SN. Total joint arthroplasty in the treatment of advanced stages of thumb carpometacarpal joint osteoarthritis. J Hand Surg [Am]. 2006;31(10):1605-14.

29. de la Caffinieri JY, Aucouturier P. Trapezio-metacarpal arthroplasty by total prosthesis. Hand. 1979;11(1):41-6.

30. Eaton RG, Glickel SZ. Trapeziometacarpal osteoarthritis. Staging as a rationale for treatment. Hand Clin. 1987;3(4):45571.

31. Jester A, Harth A, Wind G, Germann G, Sauerbier M. Disabilities of the arm, shoulder and hand (DASH) questionnaire: Determining functional activity profiles in patients with upper extremity disorders. J Hand Surg [Br]. 2005;30(1):23-8.

32. Conolly WB, Rath S. Revision procedures for complications of surgery for osteoarthritis of the carpometacarpal joint of the thumb. J Hand Surg [Br]. 1993;18(4):533-9. 